\title{
OSTI \\ AN APPLICATION OF ROBUST PARAMETER DESIGN USING AN ALTERNATIVE TO TAGUCHI METHODS
}

\author{
Marcey L. Abate, Sandia National Laboratories, Michael C. Morrow, Eastman Chemical Company, \\ and Thomas Kuczek, Purdue University \\ Marcey L. Abate, Sandia National Laboratories*, Albuquerque, NM 87185-0829 \\ *This work was supported by the United States Department of Energy under Contract DE-AC04-94AL85000
}

Key Words: Bootstrap, Noise Factors, Optimality, Robust Product Design

\section{Background}

In designing a product or process, it is often necessary to take many different types of factors into consideration. However, the factors of interest can generally be classified into two categories, controllable and uncontrollable. Controllable (or control) factors represent those factors which can be regulated. Examples of control factors include the choice of material, flow rates, processing pressures, times, and temperatures. Uncontrollable (noise) factors are factors that are either difficult, impossible, or too expensive to control during actual production or use. Examples of noise factors are environmental conditions such as ambient temperature or humidity, process parameters which are dictated by an outside source such as end user demand, and usage factors such as how long and at what temperature a consumer stores a product. It has been recognized that these noise factors may cause product characteristics to deviate from an established target value. If the noise factors transmitting the greatest amount of variation could be identified and controlled, perhaps the product characteristic could remain on target. However, by definition, the noise factors cannot be controlled in actual use or production. Thus, in order to reduce the effect of the noise factors on the product characteristic, values should be selected for the control factors such that sensitivity to the noise factors is reduced. A reduction in sensitivity is often measured in terms of the product characteristic being on target with minimal variability. A product designed in this manner is said to have a robust design.

\section{Taguchi's Approach}

One of the individuals primarily responsible for bringing the idea of robust parameter design to the attention of modern manufacturing is Dr. Genichi Taguchi. He deserves recognition for not only vigorously promoting the idea of robust product designs but also for showing that experimental design can be used as a formal part of the engineering process to accomplish a robust design. Because the noise

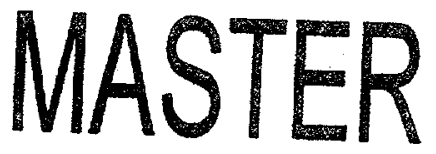

DISTRIBUTION OF THIS factors cannot be controlled. Taguchi advocated the use of experimental design to identify the settings of the controllable factors such that the effect of the noise factors is minimized

A typical Taguchi type experiment consists of a design matrix or inner array which specifies the settings of the control factors and is usually a fractional or screening design. It is assumed for the purpose of experimentation, that the noise factors can be temporarily fixed in order to assess their effect on the product characteristic. The different combinations of temporarily fixed noise factor settings are specified in a noise matrix or outer array which is usually a $2^{k}$ design. The two arrays are then crossed, so that every combination of control factors in the inner array takes place at every condition called for by the outer array of noise factors. The purpose of crossing the arrays is to deliberately induce variation due to the noise factors in a balanced manner. A response is then measured at every "design point" of the crossed array.

A typical Taguchi type analysis would involve calculating for each combination of the control factors, a performance statistic, usually called the signal-tonoise $(S / N)$ ratio. It appears that the $S / N$ ratio is intended to be a combined measure of the mean and variability of the product characteristic (Myers, et. al, 1992). The goal of the experiment is to identify the combination of control factors in the inner array which maximize the $\mathrm{S} / \mathrm{N}$ ratio. The analysis may also involve performing an ANOVA on the observed responses in order to identify which factors have an effect only on the product mean. In addition, an ANOVA may be performed on the $\mathrm{S} / \mathrm{N}$ ratio to look for variables that effect both the product variance and the mean, so that they may be used to minimize the variation. More details about typical analyses of Taguchi experiments are given in Kackar (1985) and Taguchi and Wu (1980).

Taguchi's use of experimental design is somewhat novel and differs from typical experimental design approaches and so it is not surprising that his methods have been met with various criticisms. Articles by Box (1985, 1988), Easterling (1985), Pignatiello and Ramberg (1985), and Myers et al. (1992) all explicitly discuss some common criticisms. The most dominant are (1) inefficiency in terms of the potential size of

This work was supported by the United States Department of Energy under Contract DE-AC04-94AL85000.

Sandia is a multiprogram laboratory operated by Sandia Corporation, a Lockheed Martin Company, for the United States Department of Energy. 


\section{DISCLAIMER}

Portions of this document may be illegible in electronic image products. Images are produced from the best available original document. 
experiments, (2) lack of flexibility in modeling, (3) the signal-to-noise ratio, and (4) preoccupation with optimization.

In reference to criticisms (1) and (2), a very large number of experimental trials may be required because the noise matrix is repeated for every row in the design matrix. In addition, designs suggested by Taguchi are not flexible with regard to modeling because they specify the treatment combinations that are run in both the design and the noise matrices, thus dictating the estimation of certain effects regardless of their importance. In addition, Taguchi designs often assume that the control factors do not interact (Myers et al. 1989). Therefore, one cannot use engineering knowledge or results of previous experimentation in choosing to include or to ignore specific interactions. Together, the previous can result in inflexible and unnecessarily large experiments.

Dealing with criticism (3), as Easterling (1985) points out, the collapsing of the data to $\mathrm{S} / \mathrm{N}$ ratios throws away useful information. It masks the specific relationships between the control and noise factors by aggregating over all the noise factors in the calculation of the $S / N$ ratio. The literature abounds with lively discussions and alternatives to the signal to noise ratio (Box 1988, Easterling 1985, Leon, Shoemaker, and Kackar 1987, Pignatiello and Ramberg 1985, Welch, Yu, Kang, and Sachs 1990). Lastly in regards to criticism (4), the preoccupation with optimization, the Taguchi experiments have traditionally been used to only pick a single winning combination of control factor settings. This can be problematic when because of cost differences between different control factor settings, one may wish to consider a region of suitable settings. In addition, the Taguchi methods identify the best combination of control factor settings observed in the experiment. If the control factor is a continuous variable, perhaps a more desirable setting occurs at a combination not directly observed in the experiment. By only optimizing a single criterion, a sacrifice is made in regards to potentially valuable knowledge about the product.

\section{A Response Surface Alternative}

Given these criticisms, a more desirable experimentation method would provide greater flexibility in the design of the experiment, utilize a more meaningful performance statistic, and lend itself to a better understanding of the product or process. One such experimentation method which has been proposed in the recent literature (Lucas 1994, Shoemaker et al. 1991, and Welch et al. 1990) is a response surface alternative which includes both the control factors and the noise factors in the design matrix. By fixing the noise factors for the purpose of experimentation, the response surface methodology provides a basis for estimating the product characteristic as a function of both the control and noise factors. Given this relationship, the behavior of the characteristic can be estimated when the noise factors are truly random by assuming the product experiences a random set of noise factors and applying the appropriate mean and covariance operators. Therefore, the product characteristic mean and variance are given not by hypothesizing a model for the mean and variance (as in Vining and Meyers (1990)), but by manipulation of a model for the product characteristic which assumes the noise and control factors are both fixed. For example, suppose the response product characteristic can be expressed as a second order polynomial function of a set of $k$ control factors, denoted by $\left(x_{1}, x_{2}, \ldots, x_{k}\right)$, and a set of $p$ noise factors, denoted by $\left(z_{1}, z_{2}, \ldots, z_{p}\right)$. In matrix notation, such a second order response surface for the product characteristic of interest would be given by:

$$
\mathrm{Y}_{\mathrm{x} z}=\beta_{0}+\mathrm{x}^{\prime} \beta+\mathrm{x}^{\prime} \mathbf{B} \mathrm{x}+\mathrm{z}^{\prime} \gamma+\mathrm{z}^{\prime} \mathbf{C} \mathrm{z}+\mathrm{z}^{\prime} \mathbf{D} \mathrm{x}+\varepsilon,
$$

where $\mathrm{Y}_{\mathrm{xz}}$ denotes the product characteristic of interest as a function of a fixed set of noise and control factors, $\mathrm{x}$ is the $k$-vector of control factors, and $\mathrm{z}$ is the $p$-vector of noise factors. In order to employ classical least squares theory, it is usually assumed that $e$, a random error component, follows a normal distribution with mean zero and variance $\sigma_{\varepsilon}^{2}$. Furthermore, it is assumed that the noise factors are independent of the random error components. The coefficients $\beta_{0}, \beta, \mathbf{B}$, $\gamma, \mathbf{C}$, and $\mathbf{D}$ in the linear model are assumed fixed but unknown.

Applying the expectation operator over all sources of randomness results in the product mean, denoted by $\bar{Y}_{\mathrm{X}}$, being given by:

$$
\begin{aligned}
\overline{\mathrm{Y}}_{\mathrm{x}}= & \mathrm{E}\left(\mathrm{Y}_{\mathrm{xz}}\right)=\beta_{0}+\mathrm{x}^{\prime} \beta+\mathrm{x}^{\prime} \mathbf{B} \mathrm{x} \\
& +\mu_{\mathrm{z}}^{\prime}(\gamma+\mathbf{D x})+\mu_{\mathrm{x}}^{\prime} \mathbf{C} \mu_{\mathrm{z}}+\operatorname{tr}(\mathbf{A C}),
\end{aligned}
$$

where $\mu_{z}$ is the mean vector of the noise factors, $\mathbf{A}$ is the covariance matrix of the noise factors, and $\operatorname{tr}(\mathbf{A C})$ denotes the trace of the matrix product of $\mathbf{A}$ and $\mathbf{C}$. With this expression, one could identify the product settings which place the product characteristic on target.

Similarly, the product variance can be identified by applying the variance operator to the linear model. Because of covariance terms, the expression for the product variance is a bit more complicated to derive then the product mean. Abate (1995) shows that the product variance, denoted by $V(x)$, is given by: 


$$
\begin{aligned}
\mathrm{V}(\mathrm{x})= & \mathrm{K}+\mathrm{x}^{\prime} \mathbf{D}^{\prime} \mathbf{A D} \mathrm{D}+2 \mathrm{x}^{\prime} \mathbf{D}^{\prime}\left(\mathbf{A} \gamma+\mathrm{E}\left(\mathrm{zz^{ \prime }} \otimes \mathrm{z}^{\prime}\right) \operatorname{vec}(\mathbf{C})\right) \\
& -2 \mu_{\mathrm{z}}^{\prime} \mathbf{D x}\left(\operatorname{tr}(\mathbf{A C})+\mu_{z}^{\prime} \mathbf{C} \mu_{z}\right)
\end{aligned}
$$

where $\mathrm{K}$ denotes a constant in $\mathrm{x}, \operatorname{vec}(\mathbf{C})$ is defined to be the vector formed by stacking the columns of $\mathbf{C}$ on top of each other, and $\mathbf{A} \otimes \mathbf{B}$ is defined to be the Kronecker product of the matrices $\mathbf{A}$ and $\mathbf{B}$. Therefore, in order to completely specify the product mean and variance, moments of the noise distribution must be identified. In particular, the mean vector, the covariance matrix, and possible some higher order moments and joint moments may have to be specified.

The derivations for the product mean and variance were done without restrictions on the parametric distribution of the noise factors. The current literature has derived the mean and variance response surfaces only under the restrictive assumption that the noise factors are uncorrelated and uniform over the design region (Box and Jones 1992, Vining, et al. 1992) or normally distributed over the design region (Evans 1995). While such assumptions on the noise distributions considerably simplify the derivation of the process mean and variance they may be unrealistic. For example, suppose the noise factors of interest were ambient temperature and humidity. It does not seem sensible to consider either of these to be uniform and such noise factors would almost certainly be correlated. It is also easy to envision noise factors that do not follow a normal distribution. For example, it is expected that noise factors such as storage time of food or other products which may deteriorate will follow a skewed distribution. With this in mind, the general expressions derived for the process mean and variance are preferable and more flexible then those available in the current literature. Given these two expressions, the related response surfaces for the product mean and variance could be explored graphically for a region of the control factor settings such that the product characteristic is near the target value with minimal variability. When the number of control factors would lead to complicated graphical exploration, it may be more clear to consider a single meaningful criteria. Such a criteria, which encompasses the two notions of being on target with minimum variance is mean squared error loss, defined by:

$$
\begin{aligned}
L(x) & =E\left[Y_{x x}-T\right]^{2} \\
& =E\left[Y_{x z}-\bar{Y}_{x}\right]^{2}+\left[\bar{Y}_{x}-T\right]^{2} \\
& =V(x)+B(x)^{2},
\end{aligned}
$$

where $T$ represents the specified target value, $B(x)$ is the bias, and $V(x)$ is the product variance as defined previously. The expectation is taken over the probability density function of the noise factors. Thus,
$L(x)$ is a combined measure of the product variance and distance to target. Minimizing the loss function over the control factors will identify settings which simultaneously depend on the bias and variance.

\section{A Case Study}

This response surface alternative to robust parameter design was applied to a plastic pellet manufacturing process at Eastman Chemical Company. A major goal in the production of plastic pellets is to keep a product characteristic, denoted $Y$, close to a target value of 29.996. Substandard or unsellable product may result if the characteristic deviates far enough from the established target value. The Eastman engineers knew that many inputs affected the product characteristic of interest. In particular, they had identified one uncontrollable factor, denoted $z_{1}$, and two control factors, $x_{1}$ and $x_{2}$, which influenced the value of the characteristic, Y. In order to better understand how these factors are related to the product characteristic, a three factor Box-Behnken design was run on the process. A Box-Behnken design was chosen in order to allow for the estimation of curvature effects because it was felt that running the process at the extreme vertices of a factorial design would not be possible. Three replicates were added at the center point conditions to give an estimate of pure error and to track the process stability during the experiment. The resulting experimental data is given in Figure 1, which shows the observations taken at the given values of the two control factors, $x_{1}$ and $x_{2}$. Multiple observations at each $\left(\mathrm{x}_{1}, \mathrm{x}_{2}\right)$ combination correspond to different values of $z_{1}$ with the exception of the center point replication where $z_{1}=0$.

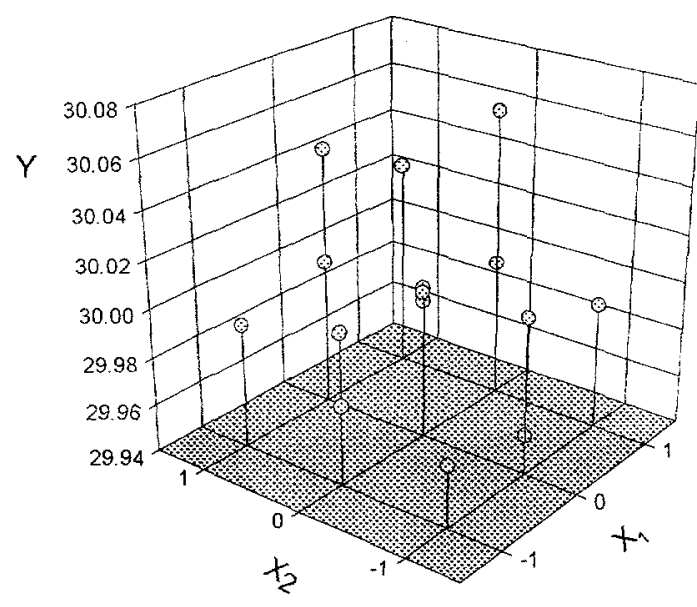

Figure 1: Experimental Data. 
A least squares fit of the data was made. The resulting estimated response surface model contained linear, quadratic, and interaction terms. The lack of fit was found to be insignificant and diagnostics did not reveal any departures from model assumptions. The reduced model that was eventually adopted on the basis of the analysis was:

$$
\begin{aligned}
\hat{\mathrm{Y}}_{\mathrm{xz}}= & 30.002+.018 x_{1}+.019 x_{2}-.008 x_{2}^{2} \\
& -.022 z_{1}-.009 x_{1} z_{1}+.01 z_{1}^{2}
\end{aligned}
$$

The estimated coefficients in the previous equation can be used to generate an estimated response surface for the mean and variance of $Y$. By taking the expectation and variance over the noise factor, the estimated mean model is given by:

$$
\begin{gathered}
\hat{\bar{Y}}_{\mathrm{x}}=30.002+.018 x_{1}+.019 x_{2}-.008 x_{2}^{2} \\
-\left(.022+.009 x_{1}\right) E\left(z_{1}\right)+.01 E\left(z_{1}^{2}\right)
\end{gathered}
$$

and the estimated variance by:

$$
\begin{aligned}
\operatorname{Vâr}(\mathbf{x}) & =\mathrm{K}+\left(.022+.009 x_{1}\right)^{2} \operatorname{Var}\left(z_{1}\right) \\
& -\left(.0002 x_{1}\right)\left(E\left(z_{1}^{3}\right)-E\left(z_{l}\right) E\left(z_{1}^{2}\right)\right)
\end{aligned}
$$

Notice that the estimated response surface for the variance is only a function of $x_{1}$. This is because the control factor $\mathrm{x}_{2}$ does not interact with the noise factor, and therefore does not have a significant effect on the variance of $\mathrm{Y}$. In order to identify the optimal settings of the control factors, it is necessary to specify the first through third moments of the noise factor. A historical data set was available that gave the values of the noise factor over an eight month period following the experiment. Production constraints throughout these eight months resulted in the historical noise factor levels being on the high side and often outside the region of the experimental design. Figure 2 is a histogram of the historical noise data which were used to estimate the moments necessary to completely specify the estimated mean and variance response surfaces.

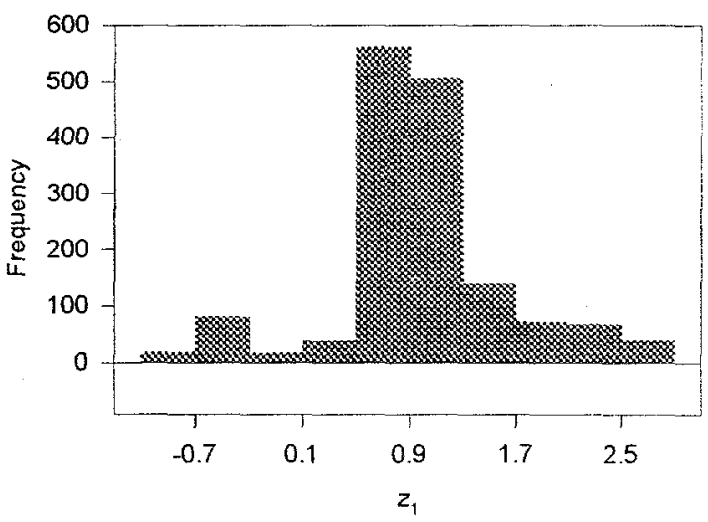

Figure 2: Histogram of the Historical Noise Data for $z_{1}$.
Substituting the estimated moments for $z_{1}$ into the estimated product mean yields the response surface in Figure 3 and shows that the target value of 29.996 can be achieved for $x_{1}$ ranging between -1 and 1 and corresponding $x_{2}$ values between 0 and 1 . Figure 4 gives the estimated standard deviation for $\mathrm{Y}$ (actually the square root of the variance plus a constant) and has a discernible minima around -.5 .

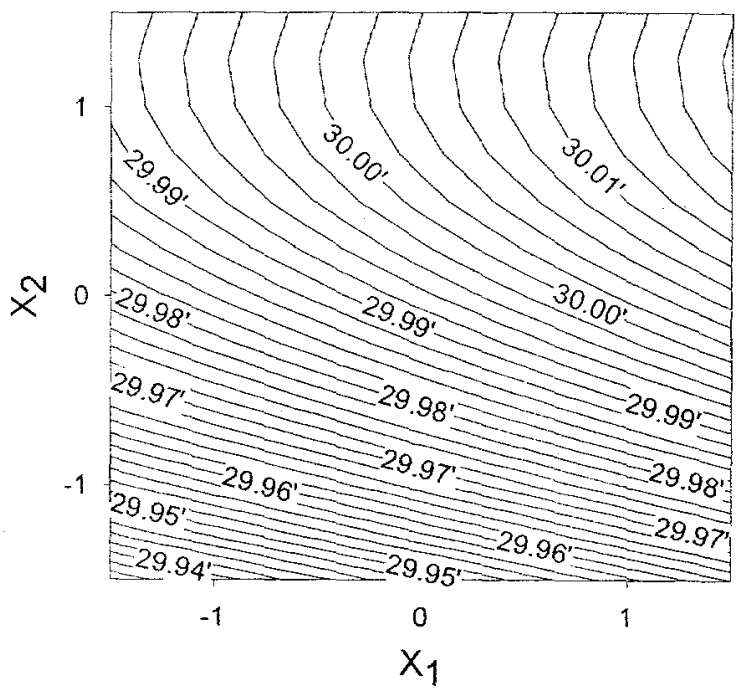

Figure 3: Estimated Product Mean Response Surface.

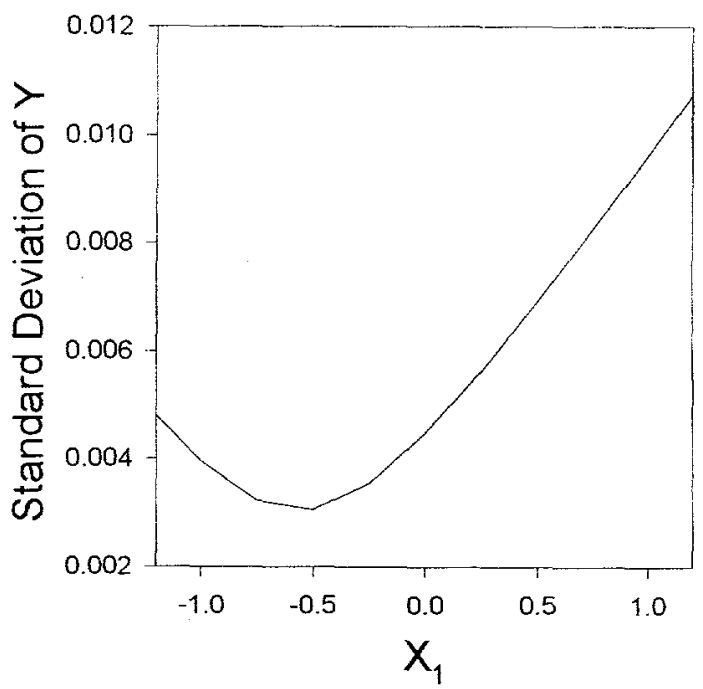

Figure 4 : Estimated Product Variance Response Surface.

Using the estimated mean and standard deviation response surfaces, the corresponding estimated optimal settings found by minimizing the loss function are $x_{1}=$ -.550 and $x_{2}=.533$. Considering Figures 3 and 4 , these minima of the loss function are not surprising. However, investigation of these figures also reveals that there may be a larger range of optimal control 
factor settings which place the response nearly on target with minimal variance. In addition, these estimated optimal control factor settings are random variables because they are functions of random least squares estimates and estimated historical noise factor moments. That is, both the experimental and historical data transmit variability to the estimated optimal settings. The experimental data transmits variability through the least squares estimates, and the historical data through the estimated noise factor moments. For these reasons, it was desired to assess the variability associated with the estimated optimal control factor settings. Because only the data at hand is available to assess this variability, a resampling method was applied.

To assess the variability associated with the use of historical data in estimating the noise factor moments, a non-parametric bootstrap technique was utilized. In this technique, B resamples are drawn with replacement from the original collection of observed noise factor values. This results in $\mathrm{B}$ resampled noise factor data sets. From each of these resampled noise factor data sets, the moments necessary to specify the estimated product mean and variance are computed. This exercise results in estimates of the noise factor moment distributions.

The other source of variability associated with the estimated optimal settings arises from using the experimental data to estimate the response surface parameters. The assumptions associated with the use of the response surface model imply that the underlying source of variability associated with the experimental data is the error term distribution which is assumed to be normal with zero mean and constant variance. In this experiment, the center points were used to estimate this variance and thus the pure error distribution. From this parametric distribution, $B$ resamples of error terms were simulated. Using these B sets of error terms, the settings of the control factors and noise factors from the original Box-Behnken design, and the original least squares estimates, B sets of experimental data were constructed. For each of these $B$ sets of experimental data, the response surface model parameters were again estimated. Use of this parametric bootstrap results in estimates of the least squares distributions.

Combining the non-parametric and the parametric bootstrap methods previously outlined, B sets of both the historical noise moments and the response surface least squares estimates are generated. Each set of these resampled estimates may then be substituted into the expressions for the product mean and variance in order to form the loss function. Minimizing the loss function for each set of resample estimates results in B estimates of the optimal control factor settings. By calculating the standard error across these B estimates, a statement of variability associated with the optimal settings may be obtained. Denoting the estimated parametric error distribution by $N\left(0, \sigma_{\varepsilon}^{2}\right)$, and the original vector of historical noise data by $\vec{h}$, this dual bootstrap procedure can be pictured as in Figure 5. Applying this dual bootstrap procedure with $\mathrm{B}=1000$ resamples results in the estimated optimal settings and associated standard errors given in Table 1.

Table 1: Estimated Optimal Settings and Standard Errors.

\begin{tabular}{|c|c|}
\hline$\hat{x}_{1}=-.550$ & $\hat{s} e\left(\hat{x}_{1}\right)=.263$ \\
\hline$\hat{x}_{2}=.533$ & $\hat{s} e\left(\hat{x}_{2}\right)=.412$ \\
\hline
\end{tabular}

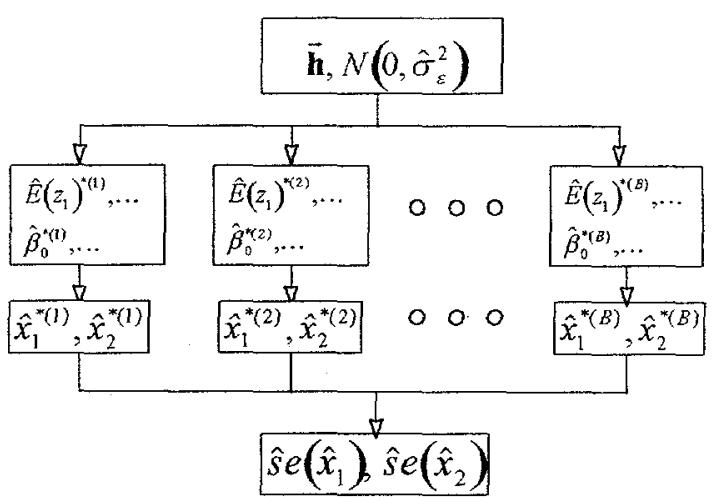

Figure 5: Dual Bootstrap Method.

Using the results in Table 1 , a region of possible optimal settings can be identified by considering some multiple of the standard error. For example, taking plus and minus 1.5 standard errors from the estimated optimal settings results in consideration of the region bounded by:

$$
-.945 \leq x_{1} \leq-.156 \text { and }-.085 \leq x_{2} \leq 1.151 \text {. }
$$

Such a region may be used for further confirmatory experimentation. Comparing this region back to Figures 3 and 4 of the product mean and variance, it is obvious that it corresponds to control factor settings where the estimated mean response was very close to the target value of 29.996 with minimal variance. Thus, in this case study, graphically exploring the product mean and variance leads to a conclusion similar to that found by formally 
minimizing an appropriate loss function and assessing the associated variability. The usefulness of this more formal process is most apparent in situations where the number of control factors complicate strict graphical exploration of the product mean and variance response surfaces. By applying the response surface methodology demonstrated in this case study, control factor settings were identified which placed the characteristic of interest on target with minimal variability, thus resulting in a robust parameter design. In addition, use of this response surface alternative to Taguchi methods lent itself to a better understanding of the manufacturing process

\section{References}

1. Abate, Marcey L., (1995), "The Use of Historical Data in Statistical Selection and Robust Product Design," Ph.D. Thesis, Department of Statistics, Purdue University.

2. Box, George E. P. (1985), "Discussion of Off-Line Quality Control, Parameter Design, and the Taguchi Methods," Journal of Quality Technology, 17, 198-206.

3. Box, George E. P. (1988), "Signal-to-Noise Ratios, Performance Criteria, and Transformations,". Technometrics, $30,1-17$.

4. Box, George E. P., and Jones, Stephen P. (1992), "Designing Products That are Robust to the Environment," Total Quality Management, 3, 265-282.

5. Easterling, Robert G. (1985), "Discussion of OffLine Quality Control, Parameter Design, and the Taguchi Methods," Journal of Quality Technology, 17, 198-206

6. Evans, Bradley P. (1995), "Some Improvements to Taguchi Analysis", Ph.D. Thesis, Department of Statistics, Purdue University.

7. Kackar, Raghu N. (1985), "Off-Line Quality Control, Parameter Design, and the Taguchi Methods," Journal of Quality Technology, 17, 198-206.

8. Leon, Ramon V., Shoemaker, Anne C, and Kacker, Raghu, N. (1987), "Performance Measures Independent of Adjustment ," Technometrics, 29, 253-265.
9. Lucas, James M. (1994). "How to Achieve a Robust Process Using Response Surface Methodology," Journal of Quality Technology, 26, 248-260

10. Myers, Raymond H., Khuri, Andre I., and Vining, Geoffrey (1992), "Response Surface Alternatives to the Taguchi Robust Parameter Design Approach," The American Statistician, 46, 131139

11. Myers, Raymond H., Khuri, Andre I., and Carter, Walter H. (1989), "Response Surface Methodology: 1966-1988," Technometrics, 31, 137-157.

12. Pignatiello, Joseph J., and Ramberg John, S. (1985), "Discussion of Off-Line Quality Control, Parameter Design, and the Taguchi Methods," Journal of Quality Technology, 17, 198-206.

13. Shoemaker, Anne C., Tsui, Kwok-Leung, and Wu, C. F. Jeff (1991), "Economical Experimentation Methods for Robust Design," Technometrics, 33, 415-428.

14. Taguchi, Genichi, and Wu., Y. (1980), Introduction to Off-Line Quality Control, Central Japan Quality Control Association, Nagoya, Japan.

15. Vining, Geoffrey G., and Myers, Raymond $H$. (1990), "Combining Taguchi and Response Surface Philosophies: A Dual Response Approach," Journal of Quality Technology, 22, 38-45.

16. Welch, William J., Yu, Tat-Kwan, Kang, Sung Mo, and Sacks, Jerome (1990), "Computer Experiments for Quality Control by Parameter Design," Journal of Quality Technology, 22, 1522. 